\author{
Poul Erik Petersen \\ World Health Organization, Oral Health Programme, Geneva, Switzerland
}

\title{
Summary
}

Chronic diseases and injuries are overtaking communicable diseases as the leading health problems in all but a few parts of the world. This rapidly changing global disease pattern is closely linked to changing lifestyles which include diet rich in sugars, widespread use of tobacco and increased consumption of alcohol. These lifestyle factors also significantly impact oral health, and oral diseases qualify as major public health problems owing to their high prevalence and incidence in all regions of the world. Like all diseases, they affect primarily the disadvantaged and socially marginalized populations, causing severe pain and suffering, impairing functionability and impacting quality of life. Traditional treatment of oral diseases is extremely costly even in industrialized countries and is unaffordable in most low and middle-income countries. The WHO Global Strategy for prevention and control of noncommunicable diseases and the "common risk factor approach" offer new ways of managing the prevention and control of oral diseases. This report outlines major characteristics of the current oral health situation in Africa and development trends as well as WHO strategies and approaches for better oral health in the $21^{\text {st }}$ century.

Key words: Africa, oral health, risk factor, development

\section{THE BURDEN OF ORAL DISEASE}

Despite vast improvement in global oral health, problems still persist in many communities and populations around the world - particularly among the underprivileged in both developed and developing countries.

\footnotetext{
Correspondence: Dr Poul Erik Petersen

World Health Organization, Oral Health

Programme, Chronic Disease and Health

Promotion, 20 Avenue Appia, 1211 Geneva 27,

Switzerland.petersenpe@who.int
}

Http:// www.ajoh.org

The distribution and severity of oral diseases vary in different parts of the world and within the same country or region. Several oral epidemiological studies have been carried out applying WHO methodology and criteria ${ }^{1}$. Currently, the dental caries level is high in several American and European countries, while it appears less severe in most African countries. Figure 1 highlights the dental caries experience among 12-year-old children according to the six WHO regional offices in the year $2000^{2,3}$, based on the DMFT (Decayed, Missing and Filled Teeth index.

Missing and Filled Teeth index.

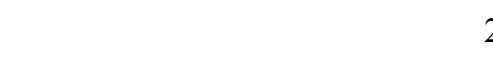


Figure 1 Dental caries experience (DMFT) of 12-year-old children according to WHO regional offices (Source: WHO Global Oral Health Data Bank and WHO Oral Health Country/Area Profile Programme, 20002,3

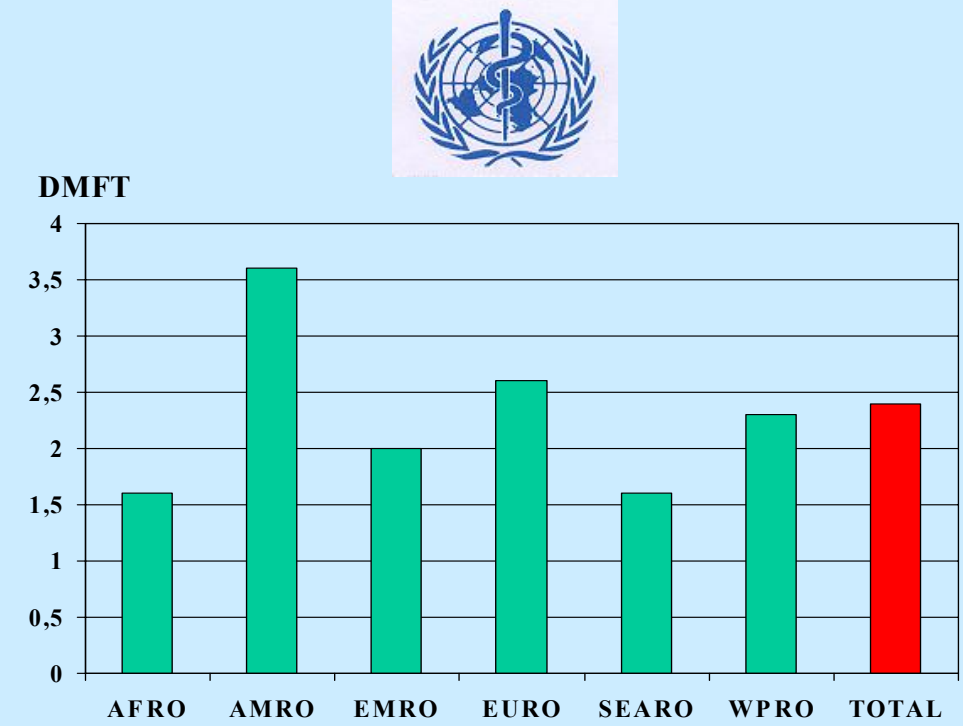

Figures 2-3 outline the current levels of dental caries in 12-year-olds and 35-44 year olds in Africa. However, the incidence of dental caries is predicted to increase in several developing countries in the region, particularly as a result of a growing consumption of sugars and inadequate exposure to fluorides.

In many African countries, access to oral health services is limited and teeth are often left untreated or are extracted to relieve pain or discomfort. This is reflected in the components of the DMFT index as shown for some AFRO countries $^{4-7}$ in Figure 4. Losing teeth is still seen by many as a natural consequence of ageing. Some countries have in recent years experienced an increase in tooth loss among adults in Africa, the proportion of edentulous adults aged 65 years varies from $6 \%$ in Gambia $^{2}$ to $25 \%$ in Madagascar ${ }^{4}$.
Figure 2. Dental caries levels (DMFT) of 12-year-olds in Africa ${ }^{2,3}$

Denntal caries levels (DMFT) of 12-year-olds, Africa

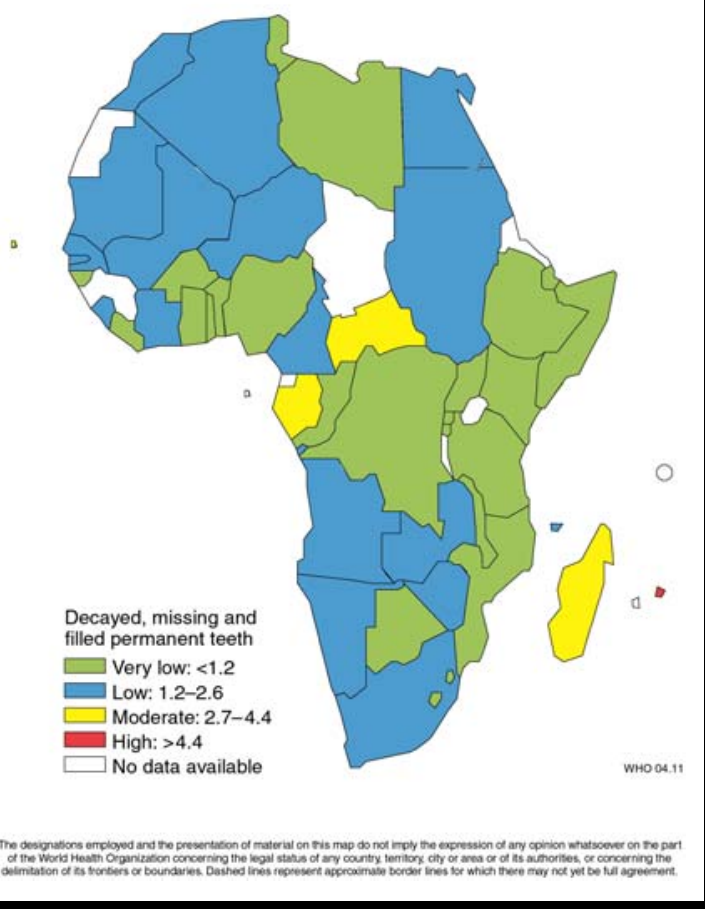




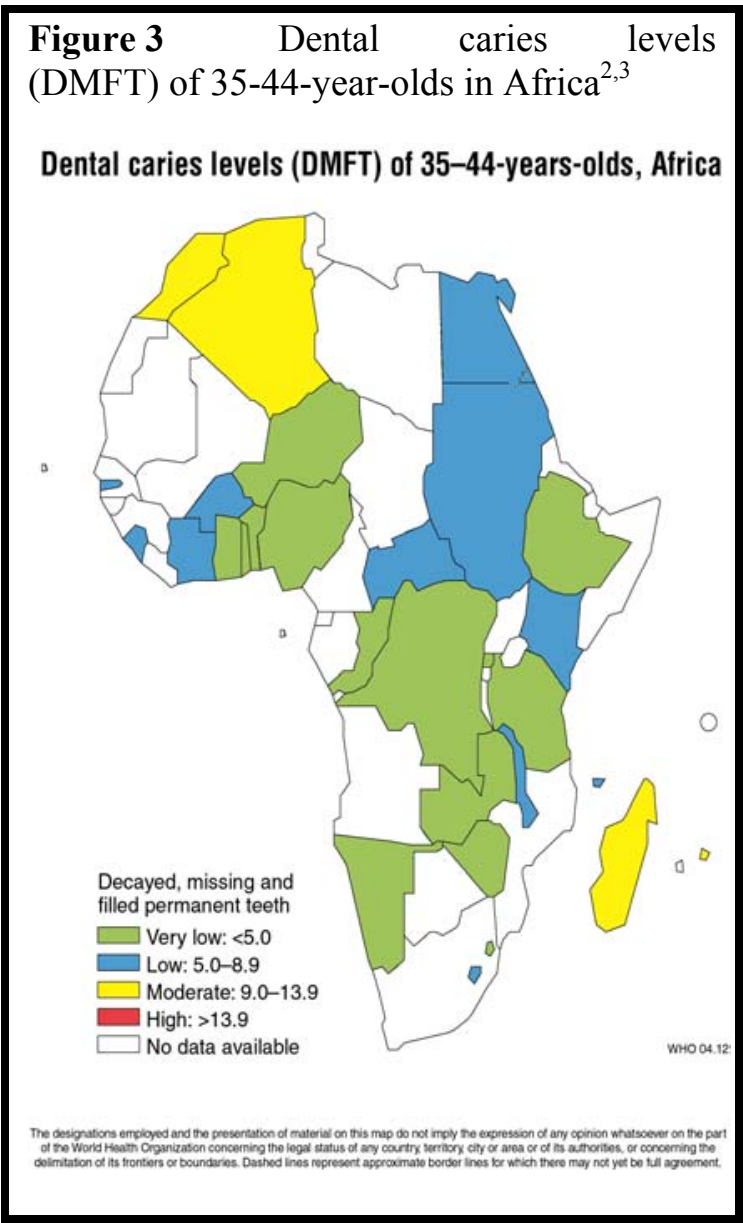

Globally, most children have signs of gingivitis and, among adults, the initial stages of periodontal diseases are prevalent. Severe periodontitis, which may cause tooth loss, is found in $5-15 \%$ of most populations. The periodontal conditions of populations surveyed recently in some African countries ${ }^{4-7}$ are highlighted in Figure 5; the surveys used the so-called Community Periodontal Index ${ }^{1}$.

The prevalence of oral cancer is particularly high among the male population, and is the eighth most common cancer worldwide ${ }^{8}$. Figure 6 shows the figures available on oral cancer in Africa related to the oral cavity. The high incidence rates relate directly to risk behaviours such as smoking, use of smokeless tobacco and alcohol consumption. In Africa the prevalence of smoking is particularly high in men and varies from $15 \%$ of adults in Nigeria to $67 \%$ in Kenya ${ }^{9}$.

Figure 4 Dental caries experience (DMFT) of 12-year-olds in selected AFRO countries ${ }^{4-7}$
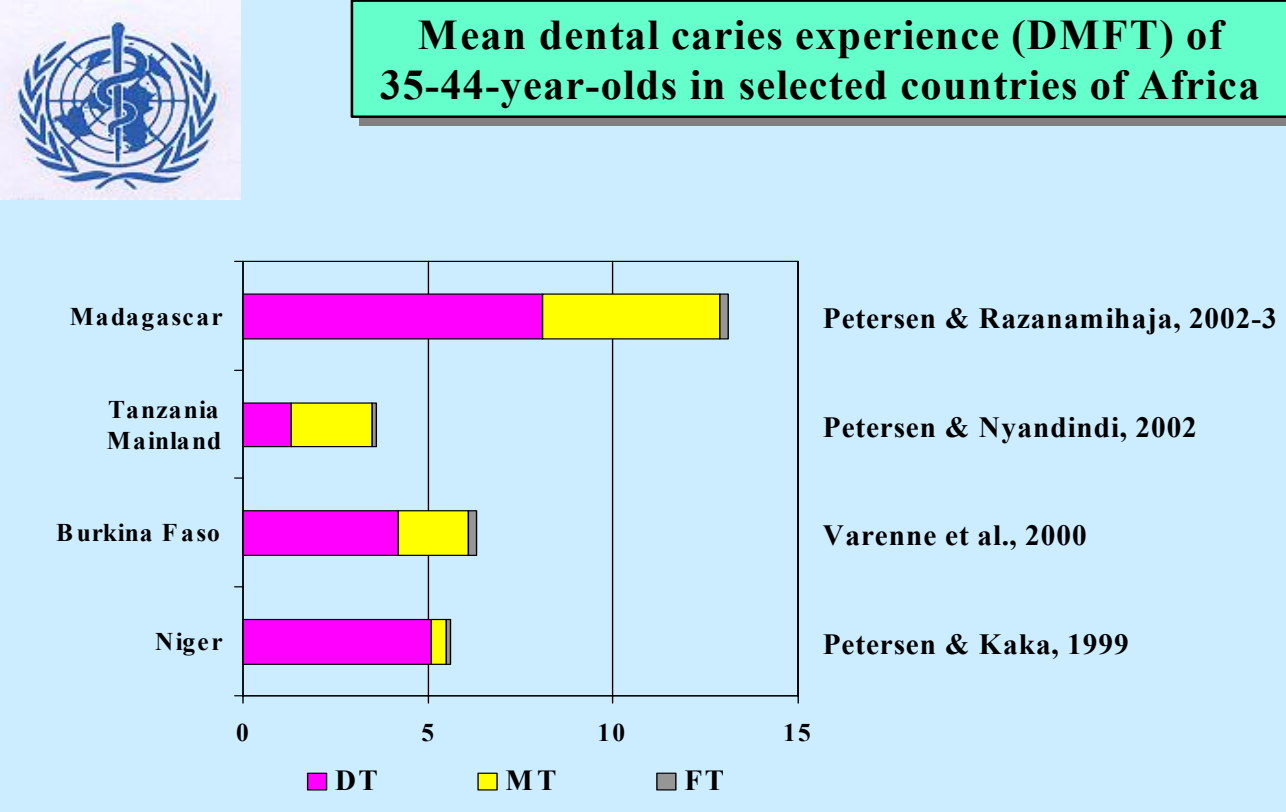

Petersen \& Razanamihaja, 2002-3

Petersen \& Nyandindi, 2002

Varenne et al., 2000

Petersen \& Kaka, 1999 
Figure 5 Mean percentages of 35-44-year-olds by maximum Community Periodontal Index scores in selected AFRO countries $^{4-7}$

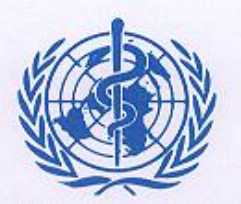

\section{Pct. of 35-44-year-olds by CPI score in selected countries of Africa}

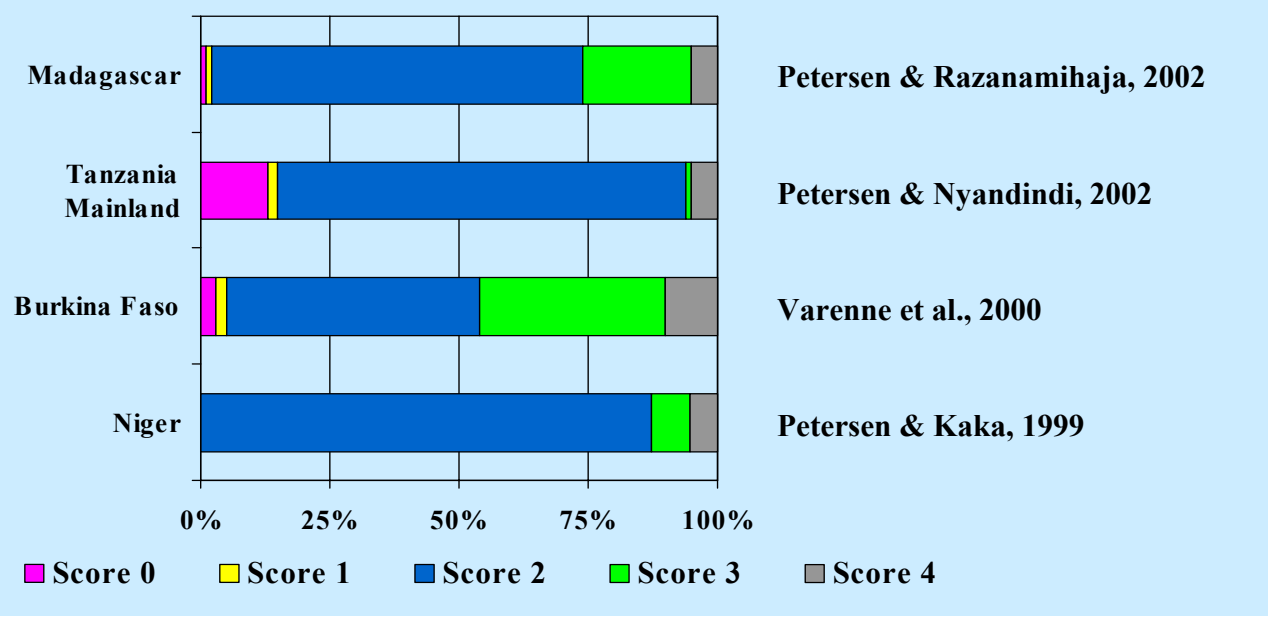

Figure 6 The incidence of cancer in countries of sub-Saharan Africa in 2000 (Source: WHO International Agency for Research on Cancer, 2003) ${ }^{8}$

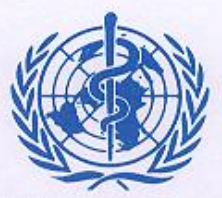

\section{Incidence of cancer in sub-Saharan Africa}

Cervix uteri

Breast

Liver

Prostate

Stomach

NHL

Colorectal

Oesophagus

Oral cavity

Male
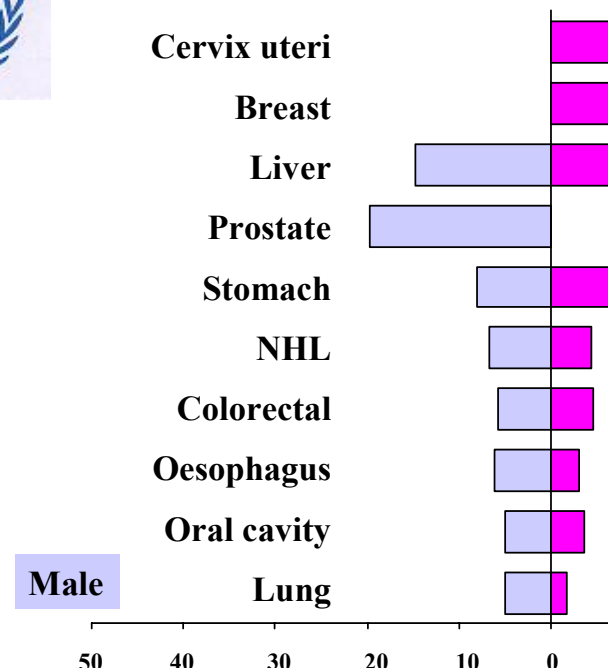

Age standardized rate / 100,000 population 
Qat is a leafy narcotic substance popular in several countries in East Africa and the Arabian Peninsula. It can be consumed in the form of tea or smoked like tobacco, although the most common mode of ingestion is by chewing the fresh leaves. Consumption of qat can lead to adverse oral effects including oral mucosal lesions, dryness of the mouth, discoloration of teeth and periodontal problems.

The regions of the world have different oral health profiles. In addition to oral precancer and oral cancer, countries in Africa must urgently address a number of very serious oral conditions including noma (cancrum oris), ANUG (acute necrotizing ulcerative gingivitis), and oral manifestations of HIV/AIDS. Analyses have shown that oral manifestations of this disease often include candida infections, hairy leukoplakia, oral ulcers and gingival bleeding, necrotizing periodontitis, leukoplakia and Kaposi's sarcoma ${ }^{3}$.

Most - particularly developing countries still lack reliable data on the frequency and severity of oro-dental trauma ${ }^{10}$. Some countries report dental trauma in about $10-15 \%$ of children, and a significant proportion of dental trauma derives from road accidents or violence and unsafe playgrounds or schools. In industrialized countries, the costs of immediate and follow-up care for dental trauma patients are high, while such information is not available in developing countries of Africa.

Diagnosis and treatment of craniofacial anomalies such as cleft lip and palate present a number of challenges to public health. Oro-facial clefts occur in around 1 per 500-700 births, the rate varying substantially across ethnic groups and

Http:// www.ajoh.org geographical areas ${ }^{11}$ and appear to be environment-related, a higher risk being associated with the mother's use of tobacco and alcohol and her nutritional level. There are many parts of the world, in particular parts of Africa for which there is little or no information available on the frequency of craniofacial anomalies. There is to date no consistent evidence of trends over time, nor is there consistent variation by socioeconomic status, but these aspects have not yet been adequately studied. Other conditions that may lead to special health care needs include Down's syndrome, cerebral palsy, learning and developmental disabilities, and genetic and hereditary disorders with oro-facial defects.

\section{Oral disease burdens and common risk factors}

Given their prevalence worldwide, oral diseases are major public health problems. Their impact on individuals and communities in terms of pain and suffering, functional impairment and reduced quality of life is considerable, and they are the fourth most expensive to treat in most industrialized countries. Were it available in many low-income countries, treatment of dental caries in children alone would exceed the total child health care budget.

A core group of modifiable risk factors is common to many chronic diseases and injuries. The four most prominent NCDs - cardiovascular diseases, diabetes, cancer and chronic obstructive pulmonary diseases - share common risk factors with oral diseases that are lifestyle-related and preventable. The greatest burden of all diseases is on the disadvantaged and socially marginalized. A major benefit of the common risk factor approach is the focus on 
improving health conditions for the whole population as well as for high risk groups, thereby reducing inequities. The WHO Global Strategy for the prevention and control of noncommunicable diseases represents a new approach to managing the prevention and control of oral diseases.

\section{WHO STRATEGIES AND APPROACHES IN ORAL DISEASE PREVENTION AND HEALTH PROMOTION}

WHO's goals are to build healthy populations and communities and to combat ill-health. Four strategic directions provide the broad framework for WHO's technical work and in relation to oral health.

1. Reducing the burden of oral disease and disability, especially in poor and marginalized populations.

2. Promoting healthy lifestyles and reducing risk factors to oral health that arise from environmental, economic, social and behavioural causes.

3. Developing oral health systems that equitably improve oral health outcomes, respond to people's legitimate demands, and are financially fair.

4. Framing policies in oral health, based on integration of oral health into national and community health programmes, and promoting oral health as an effective dimension for development policy of society.

The threat posed by noncommunicable diseases and the need to provide urgent and effective public health responses led to the formulation of a global strategy for prevention and control of these

Http:// www.ajoh.org diseases, endorsed in 2000 by the $53^{\text {rd }}$ World Health Assembly (resolution WHA 53.17). Priority is given to diseases - including oral diseases which are linked by common, preventable and lifestyle related risk factors (e.g. unhealthy diet, tobacco use).

As emphasized in the World Oral Health Report $2003^{12}$, the high relative risk of oral disease relates to sociocultural determinants such as poor living conditions, poor access to safe water or sanitary facilities, low education levels, and lack of traditions, beliefs and culture in support of oral health. Communities and countries with inappropriate exposure to fluorides also have a higher risk of dental caries. Control of oral disease depends on the availability and accessibility of oral health systems but risk reduction is only possible if services are oriented towards primary health care and prevention. In addition to the distal sociocultural and environmental factors, the model emphasizes the role of intermediate, modifiable risk behaviours, i.e. oral hygiene practices, sugars consumption (amount, frequency of intake, types) as well as tobacco use and excessive alcohol consumption.

Clinical and public health research has shown that individual, professional and community measures are effective in preventing most oral diseases ${ }^{13}$. However, optimal intervention in relation to oral disease is not universally available or affordable because of escalating costs and limited resources. This, together with insufficient focus on primary prevention of oral diseases, poses a considerable challenge particularly for developing countries of Africa, and where populations for the most part are underserved. In several African developing countries the most 
important challenge is to offer essential oral health care within the context of primary health care programmes. Such programmes should meet the basic health needs of the population, strengthen active outreach to the community, organize primary care, and ensure effective patient referral.

The major challenges of the future will be to translate knowledge and experiences of disease prevention into action programmes. The development of such programmes is particularly difficult if oral health care is not fully integrated into national or community health programmes.

\section{Health promotion and oral health}

Health promotion deals with the broader determinants of health and seeks to reduce risks through sensitive policies and actions. Promotion of health in the settings where people live, work, learn and play is clearly the most creative and cost-effective way of improving oral health and, in turn, quality of life. Increasing urbanization as well as demographic and socio-environmental changes require comprehensive oral health action. It is unlikely that improvements in oral health can be achieved by isolated interventions that target specific behaviours. The most effective, sustainable interventions combine social policy and individual action through which healthy living conditions and lifestyles are promoted.

The WHO Global Oral Health Programme provides technical and policy support needed to enable countries to integrate oral health promotion with general health promotion. The development of

Http:// www.ajoh.org programmes for oral health in targeted countries focuses on:

* Identification of health determinants; mechanisms in place to improve capacity to design and implement interventions that promote oral health.

* Implementation of communitybased demonstration projects for oral health promotion, with special reference to poor and disadvantaged population groups.

* Building capacity in planning and evaluation of national programmes for oral health promotion and evaluation of oral health promotion interventions in operation.

* Development of methods and tools to analyse the processes and outcomes of oral health promotion interventions as part of national health programmes.

* Establishment of networks and alliances to strengthen national and international actions for oral health promotion.

In accordance with WHO overall priorities, the Global Oral Health Programme has adopted the following strategic orientations and priorities for action.

\section{PRIORITY ACTION AREAS FOR GLOBAL ORAL HEALTH}

\section{Oral health and fluorides}

Fluoride is being widely used on a global scale, with much benefit. Millions of people worldwide use fluoridated toothpaste, and/or are exposed to fluoridated water or fluoridated salt or other forms of fluoride applications (clinical topical fluorides, mouthrinses, tablets/drops). However, populations in many developing countries do not have access to fluorides for practical or

(C) African Journal of Oral Health. 
Figure 7 Use of fluoridated toothpaste (Pct.) in developing countries - the example of Burkina Faso ${ }^{14}$

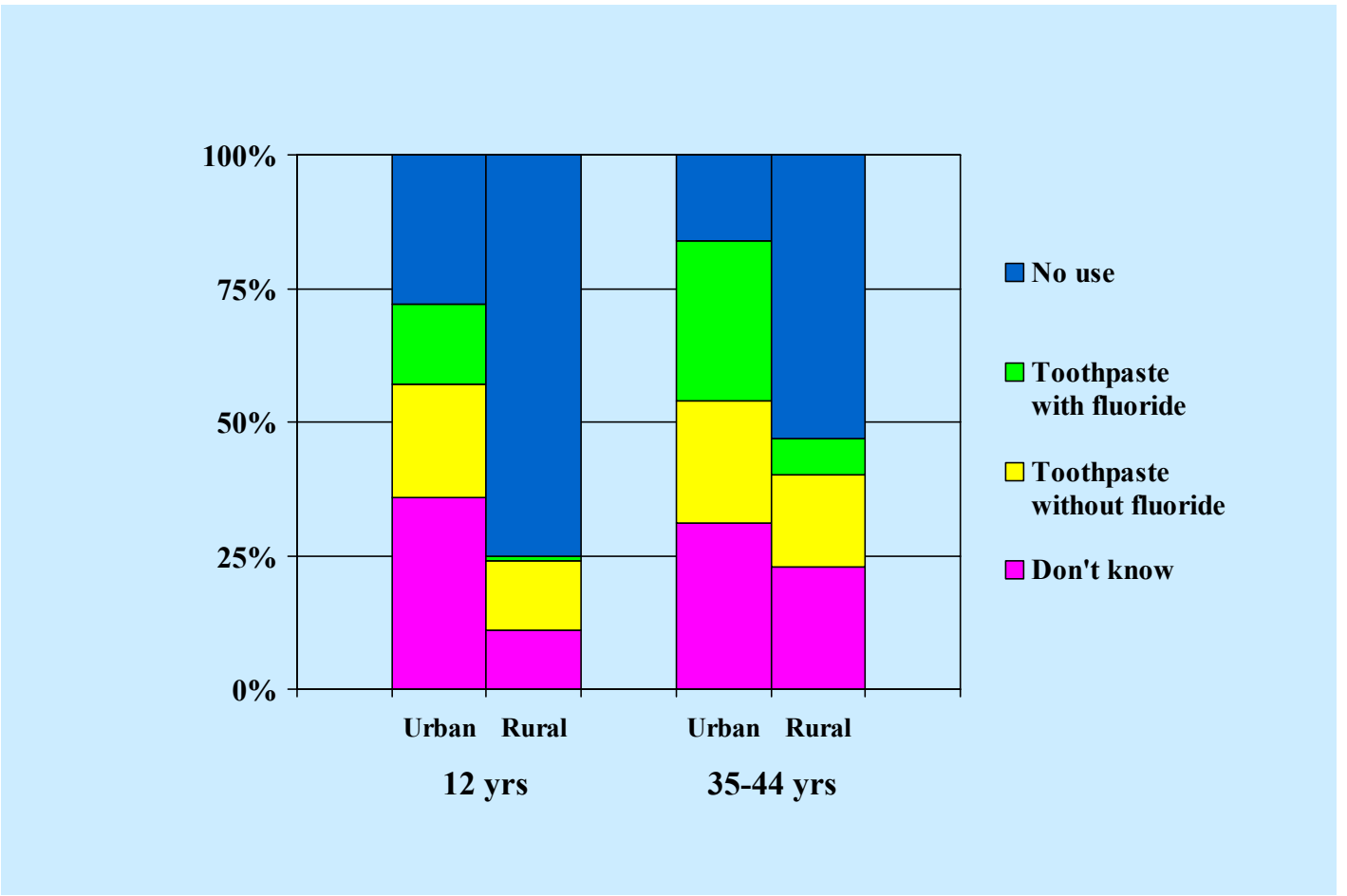

economic reasons. Experiences from Africa tell that this is particularly the case for rural populations (Figure 7) ${ }^{14}$.

In WHO Technical Report Series No. 846 on "Fluorides and oral health" $(1994)^{15}$, the recommendation on use of fluoridated toothpastes reads as follows:

Because fluoridated toothpaste is a highly effective means of caries control, every effort must be made to develop affordable fluoridated toothpastes for use in developing countries. The use of fluoride toothpastes being a public health measure, it would be in the interest of countries to exempt them from the duties and taxation applied to cosmetics.

Recommendation 12, page 36

Recent local studies have shown that affordable fluoridated toothpaste is effective in caries prevention and should be made available by health authorities in developing countries ${ }^{16}$. One of WHO's policies is to support this measure. This is particularly important in light of the changing diet and nutritional status in these countries.. The WHO Global Oral Health Programme is currently undertaking further demonstration projects in Africa in order to assess the effects of affordable fluoridated toothpaste.

\section{Diet, nutrition and oral health}

Diet and nutrition affect oral health in many ways. Nutrition, for example, influences craniofacial development, oral cancer and oral infectious diseases. Dental diseases related to diet include dental caries, developmental defects of enamel, dental erosion and periodontal disease. WHO/FAO recently published a 
Global Strategy on Diet, Physical Activity and Health ${ }^{17}$, based on analysis of the best available evidence on the relationship between diet and physical activity patterns and the major nutritionrelated chronic diseases. The strategy aims at reducing the growing burden of NCDs in both developing and developed countries. Recommendations are made to facilitate the formulation of regional strategies and national guidelines to reduce the burden of nutrition-related chronic diseases. Among other recommendations, free (added) sugars should remain below $10 \%$ of energy intake and the consumption of foods/drinks containing free sugars should be limited to a maximum of four times per day. In order to minimize the occurrence of dental erosion which seems to be closely related to the consumption of acidic beverages, the amount and frequency of intake of soft drinks and juices should be limited. Many countries currently undergoing nutrition transition do not have adequate fluoride exposure. It is the responsibility of national health authorities to ensure implementation of feasible fluoride programmes for their country.

The WHO Oral Health Programme contributes to the implementation of the Global Strategy on Diet, Physical Activity and Health. There are many intervention activities at national level but the following areas should be addressed:

* legislation and policy

* oral health services

* schools: curricula, school lunches and school health

* food industry, supermarkets

* restaurants, catering, etc.

* non-governmental organizations (NGOs) (health related and others)

* media
* monitoring, surveillance and research

The Ministries of Health should ensure that mechanisms for intersectoral collaboration are carefully considered. Strategies include taxation and pricing, food labelling, school lunch policies and support to nutrition programmes.

\section{Tobacco and oral health}

Prevalence of tobacco use has declined in some high-income countries but continues to increase in low-income and middle-income countries, especially among young people and women?. Undoubtedly, the increasing number of smokers and smokeless tobacco users among young people in some parts of the world will considerably affect the general and oral health of future generations. The prevalence of tobacco use in most countries is highest among people of low educational background as well as the poor and marginalized. In several countries of Africa the use of tobacco is higher among males than females ${ }^{9}$.

Tobacco is a risk factor for oral cancer, oral cancer recurrence, adult periodontal diseases and congenital defects such as cleft lip and palate in children. It suppresses the immune system's response to oral infection, compromises healing following oral surgery and accidental wounding, promotes periodontal degeneration in diabetics and adversely affects the cardiovascular system. Moreover, the risks of tobacco use greatly increase when it is used in combination with alcohol or areca nut. Most of the oral consequences of tobacco use impair quality of life, be they as simple as halitosis (bad breath), as complex as oral birth defects, as common as periodontal disease or as 
troublesome as complications during wound healing.

The WHO Oral Health Programme has designed several strategies to control tobacco-related oral diseases and adverse conditions $^{18}$. Oral health-related activities are integrated in the overall WHO tobacco-free initiative and the Programme encourages the adoption and implementation of WHO tobaccocessation and control policies by international and national oral health organizations. In addition, oral health professionals should strengthen their contributions to tobacco-cessation programmes.

\section{Oral health through Health Promoting Schools}

The Health Promoting School can be characterized as a school constantly strengthening its capacity as a healthy setting for living, learning and working. To help individuals and groups advocate Health Promoting Schools, WHO has produced an "Information Series on School Health". Guidelines are given on how to assist school and community leaders in improving the health and education of young people, and individual documents in the series encourage schools to address one or more important health issues. Trainingof-trainers programmes for schoolteachers are conducted to increase national capacities for integration of oral health promotion in schools.

The WHO Oral Health Programme has prepared an oral health technical document to strengthen the implementation of an oral health component of the Health Promoting Schools programme ${ }^{19}$. Strong arguments for oral health promotion through schools include the following:
* Pupils and students can be accessed during their formative years, from childhood to adolescence. These are important stages in people's lives when lifelong oral health related behaviour as well as beliefs and attitudes are being developed.

* Schools can provide a supportive environment for promoting oral health. Access to safe water, for example, may allow for general and oral hygiene programmes. Also, a safe physical environment in schools can help reduce the risk of accidents and concomitant dental trauma.

* The burden of oral disease in children is significant. Most established oral diseases are irreversible, will last for a lifetime and have an impact on quality of life and general health.

* School policies, the physical environment and education for health are essential for the attainment of oral health and control of risk behaviours, such as intake of sugary foods and drinks, tobacco use and alcohol consumption.

* Schools can provide a platform for the provision of oral health care, i.e. preventive and curative services.

\section{Oral health improvement amongst the elderly}

In many developing countries significant increases in the elderly population are expected by 2025. As people age, their susceptibility to chronic and lifethreatening diseases as well as acute infections increases, exacerbated by compromised immune systems. In addition to cancer, cardiovascular diseases, diabetes, and infections, poor 
oral health, most notably tooth loss and severe periodontal conditions, is prevalent in this age group. Barriers to oral health care among the elderly are considerable. Impaired mobility impedes access to care, particularly for those who reside in rural areas. The situation is worsened in developing countries when oral health services and domiciliary care are not available. The WHO Oral Health Programme intends to develop strategies for improved oral health of older people. Based on experiences from demonstration programmes in countries, national oral health planners are encouraged to integrate systematic oral health activities towards improved quality of life.

\section{Oral health, general health and quality of life}

Oral health is integral to general health. Those with ill health are at greater risk of oral diseases that, in turn, further complicate their overall health $^{20}$. Some general health diseases manifest in the mouth, and oral lesions may be the first signs of other life-threatening diseases such as HIV/AIDS. The experience of pain, endurance of dental abscesses, problems with eating and chewing, embarrassment about the shape of teeth or about missing, discoloured or damaged teeth can adversely affect people's daily lives and well-being. The WHO Oral Health Programme provides analysis-for-policy and analysis-ofpolicy information instrumental to the integration of oral health into national and community health programmes.

\section{Oral health systems}

More than 20 years after its widespread adoption, the strategy of Health for All through primary health care still has not been fully implemented. In many countries, national capacity and resources - human, financial and material - are still insufficient to ensure availability of and access to essential, high quality health services for individuals and populations, especially in deprived communities. In developing countries, oral health services are mostly offered from regional or central hospitals of urban centres and little, if any, importance is given to preventive or restorative dental care. Many countries have a shortage of oral health personnel and generally the capacity of the systems is limited to pain relief or emergency care. In Africa, the dentist to population ratio is approximately $1: 150000$ and significant proportions of children and adults have never seen a dentist (Table 1) ${ }^{4,7,14}$.

Table 1 Percentages of age groups 12 and 35-44 years in selected countries of Africa who saw a dentist over the past 12 months or who never have seen a dentist

\begin{tabular}{lllllll}
\hline & \multicolumn{2}{c}{ Burkina Faso $^{14}$} & \multicolumn{2}{c}{ Tanzania Mainland $^{7}$} & \multicolumn{2}{c}{ Madagascar $^{4}$} \\
& $12 \mathrm{yrs}$ & $35-44 \mathrm{yrs}$ & $12 \mathrm{yrs}$ & $35-44 \mathrm{yrs}$ & $12 \mathrm{yrs}$ & $35-44 \mathrm{yrs}$ \\
\hline Saw a dentist within the & & & & & & \\
past 12 months & 4 & 10 & 11 & 12 & 11 & 16 \\
\multirow{2}{*}{ Never seen a dentist } & 93 & 61 & 76 & 43 & 83 & 35 \\
\hline
\end{tabular}


The WHO Global Oral Health Programme supports the development of oral health services that match the needs of the country. For developing countries in particular, primary health care models for essential oral health care are encouraged and several community demonstration projects based on sociocultural conditions are supported or carried out jointly with the WHO Oral Health Programme. Oral treatment approaches have been designed ${ }^{21}$, moreover, the WHO Oral Health Programme has an oral health component for the project Integrated Management of Adolescent/Adult Illness - Guidelines for First-level Facility Health Workers in Low Resource Setting ${ }^{22}$.

\section{HIV / AIDS and oral health}

The HIV/AIDS epidemic is the fastestgrowing threat to development today and the epidemic is particularly severe in sub-Saharan Africa. The greatest challenge in responding to HIV/AIDS at present is to ensure that proven, gender sensitive strategies for prevention and care are widely implemented to a level where there will be significant impact on the epidemic. An oral health component of public health programmes can make important contributions to the early diagnosis, prevention and treatment of this disease. A number of studies have demonstrated that about $40-50 \%$ of HIVpositive persons have oral fungal, bacterial or viral infections, often occurring early in the course of the disease ${ }^{3}$. Oral lesions strongly associated with HIV infection are pseudomembranous oral candidiasis, oral hairy leukoplakia, HIV gingivitis and periodontitis, Kaposi's sarcoma, nonHodgkin lymphoma, and dry mouth owing to a decreased salivary flow.
The WHO Oral Health Programme has prepared a guide ${ }^{23}$ to provide a systematic approach to the implementation of epidemiological studies of oral conditions associated with HIV infection; to provide guidelines for the collection, analysis, reporting and dissemination of data from such studies; and to facilitate comparison of findings from different studies. It also aims to encourage oral health providers, primary health care workers and public health practitioners to make oral health status an integral part of optimum case management and of surveillance activities of the diseases associated with HIV infection.

\section{Oral health information systems, evidence for oral health policy and formulation of goals}

In 1981, WHO and the FDI World Dental Federation jointly formulated goals for oral health to be achieved by the year $2000^{12,24}$, as follows:

1. $50 \%$ of $5-6$ year-olds to be free of dental caries.

2. The global average to be no more than 3 DMFT at 12 years of age.

3. $85 \%$ of the population should have all their teeth at the age of 18 years.

4. A $50 \%$ reduction in edentulousness among 35-44-yearolds, compared with the 1982 level.

5. A $25 \%$ reduction in edentulousness at age 65 years and over, compared with the 1982 level.

6. A database system for monitoring changes in oral health to be established.

The establishment of oral health information systems remains a challenge 
for most countries of the world. The WHO Global Oral Health Programme is prepared to assist countries in their efforts to develop such systems which include health systems data additional to epidemiological indicators. At WHO, information systems are being established for the surveillance of global trends in oral disease and risk factors. The WHO Global Oral Health Data Bank compiles valuable information for monitoring the global epidemiological picture and trends over time in oral health. The WHO Global Oral Health Programme has initiated integration of the existing database with other WHO health databases and surveillance systems on risk factors ${ }^{9}$.

The formulation of new WHO goals has been initiated. WHO, FDI and IADR have jointly prepared new goals for the period up to the year $2020^{25}$. The objectives and targets have been broadened in order to cover significant indicators related to oral health and care of population groups. The global goals are primarily designed to encourage health policy-makers at regional, national and local levels to set standards for oral health in relation to pain, functional disorders, infectious diseases, oro-pharyngeal cancer, oral manifestations of HIV infection, noma, trauma, craniofacial anomalies, dental caries, developmental anomalies of teeth, periodontal disease, oral mucosal diseases, salivary gland disorders, tooth loss, health care services and health information systems. The WHO Global Oral Health Programme will support countries directly as well as through regional and country offices in their formulation of goals, targets and standards of oral health.

\section{Research for oral health}

Research is the systematic process for generating new knowledge. Advances in knowledge, however, have not yet benefited developing countries to the fullest extent possible. It has been estimated, for example, that only $10 \%$ of funding for global health research is allocated to health problems that affect $90 \%$ of the world's population ${ }^{26}$. Clear disparities in economic strength, political will, scientific resources and capabilities, and access global information networks have, in fact, widened the knowledge gap between rich and poor countries. The WHO Global Oral Health Programme contributes to the process of redressing the imbalance in the distribution of knowledge about oral health, so that the results of research will benefit everyone in a sustainable and equitable manner. As knowledge is a major vehicle for improving the health of poor people in particular, the WHO Oral Health Programme will focus on stimulating oral health research in the developed and the developing world to reduce risk factors and the burden of oral disease, and to improve oral health systems and the effectiveness of community oral health programmes. In particular, more research should be devoted to: inequity in oral health; the psychosocial implications of oral health/illness; diet, nutrition and oral health; tobacco cessation programmes; oral health general health - quality of life interrelationships; and HIV/AIDS.

The WHO Oral Health Programme intends to stimulate oral health research for, with and by developing countries in several ways, such as:

* Supporting initiatives that will strengthen research capability in developing countries so that 
research is recognized as the foundation of oral health policy.

* Encouraging oral health research training programmes at local level or based on interuniversity collaborative "sandwich" programmes.

* Providing universities in developing countries with easy access to the scientific literature within oral health and online access to scientific articles.

* Reducing the 10/90 gap in oral health research through work within the framework of the Global Forum for Health Research $^{26}$.

\section{CONCLUSION}

Oral diseases are major public health problems. In addition to socioenvironmental conditions, oral diseases are related to modifiable lifestyles. Unhealthy dietary habits, smoking and other tobacco use, alcohol consumption and poor hygiene are some of the common risk factors for many NCDs, including oral disease. The WHO Global Oral Health Programme enables effective execution of the common risk factor approach in disease prevention and health promotion. While there has been encouraging improvement in oral health in many countries over the past few decades, much work remains to be done. With many challenges ahead for Africa, it is important to build on our achievements, and on strategies that work.

This report highlights the priority areas for the WHO Global Oral Health Programme and provides a framework for implementation. WHO's work for oral health will focus on devising tools for intersectoral collaboration, community participation, supportive

Http:// www.ajoh.org policy decisions, oral health systems development, and particularly development of community-based strategies for oral disease control and promotion of oral health.

\section{REFERENCES}

1. World Health Organization. Oral Health Surveys - Basic Methods. 4th ed. Geneva: World Health Organization, 1997.

2. World Health Organization. Global Oral Health Data Bank. Geneva: World Health Organization, 2002.

3. World Health Organization. WHO Oral Health Country/Area Profile. (Available from the Internet on the Internet

from: http://www.whocollab.od.mah.se/i ndex.html).

4. Petersen PE, Razanamihaja M. Oral health status and oral health care in children and adults in Madagascar, 2002. Geneva: World Health Organization, 2003.

5. Petersen PE, Kaka M. Oral health status of children and adults in the Republic of Niger, Afria. Int Dent J 1999; 49:159-64

6. Varenne B, Petersen PE, Ouattara S. Oral health status of children and adults in urban and rural areas of Burkina Faso, Africa. Int Dent J 2004; 54 (in press)

7. Petersen PE, Nyandindi U, Kikwilu E, Mabelya L, Lembariti BS, Poulsen VJ. Oral health status and oral health behaviour of schoolchildren, teachers, and adults in Tanzania. Technical Report. Geneva: WHO, 2002

8. Steward BW, Kleihues P. World Cancer Report. Lyon: WHO International Agency for Research on Cancer, 2003. 
9. Mackay J, Eriksen M. The Tobacco Atlas. Geneva: WHO, 2002

10. Andreasen JO, Andreasen FM. Dental trauma. In: Pine C (ed). Community Oral Health. London: Elsevier Science Limited, 2002. p. 94-9.

11. World Health Organization. Global Strategies to Reduce the Health Care Burden of Craniofacial Anomalies. Geneva: World Health Organization, 2002.

12. Petersen PE. The World Oral Health Report 2003; continuous improvement of oral health in the 21st century - the approach of the WHO Global Oral Health Programme. Community Dent Oral Epidemio 2003; 31 (Suppl 1): 3-24

13. Cohen L, Gift H. (eds). Disease Prevention and Oral Health Promotion. Socio-dental Sciences in Action. Copenhagen: Munksgaard, 1995.

14. Varenne B, Petersen PE, Ouattara $\mathrm{S}$. Oral health behaviour profile of children and adults in urban and rural areas of Burkina Faso, Africa. Geneva: WHO, 2003

15. World Health Organization. Fluorides and Oral Health. WHO Technical Report Series No. 846. Geneva: World Health Organization, 1994.

16. Adyatmaka A et al. School-based Primary Preventive Programme for Children. Affordable Toothpaste as a Component in Primary Oral Health Care. Geneva: World Health Organization, 1998.

17. World Health Organization. Diet, Nutrition and the Prevention of Chronic Diseases. WHO Technical Report Series 916.
Geneva: World Health Organization, 2003.

18. Petersen PE. Tobacco and oral health - the role of the World Health Organization. Oral Health and Preventive Dentistry 2003, 1:309-15

19. World Health Organization. Oral Health Promotion Through Schools. WHO Information Series on School Health. Document 11. Geneva: World Health Organization, 2003.

20. Rohr Inglehart M, Bagramian RA. Oral Health-Related Quality of Life. Chicago: Quintessence, 2002.

21. WHO Collaborating Centre for Oral Health Care Planning and Future Scenarios. Basic Package for Oral Care. Nijmegen: University of Nijmegen, 2002.

22. World Health Organization. Extending essential care. Integrated management of adolescent and adult illness. Geneva: WHO, 2003.

23. Melnick SL et al. A Guide for Epidemiological Studies of Oral Manifestations of HIV Infection. Geneva: World Health Organization, 1993.

24. Federation Dentaire Internationale/World Health Organization. Global goals for oral health in the year 2000. International Dental Journal 1982; 23: 74-7.

25. Hobdell M, Petersen PE, Clarkson J, Johnson N. Global goals for oral health 2020. Int Dent J 2003; 53:285-288

26. Global Forum for health research. The 10/90 report on health research 2001-2002. (Available on the Internet from: http://www.globalforumhealth.org). 